\title{
Closed-Loop Control of Anaesthetic Effect
}

\author{
Santiago Torres, Juan A. Méndez, \\ Héctor Reboso, José A. Reboso and Ana León \\ Universidad de La Laguna \\ Spain
}

\section{Introduction}

The interest in automation technologies applied to anaesthesia has been grown exponentially in last decade. The main difference with other fields of automation is that the presence of a human supervisor has been never questioned. In spite of this, the use of automation tools to monitor and control the main variables during surgery notably helps the anaesthetist during surgery. The basic functions of the automation systems in anaesthesia are monitoring and control of the main variables of the process. This leads to two expected benefits. First, the anaesthetist will be freed of some routinary tasks so that he can concentrate more on the state of the patient. On the other hand, using these tools contributes to improve the global performance of the process in terms of safety, costs reduction and patient comfort.

During the surgery operation three main variables have to be regulated: hypnosis, analgesia and muscle relaxation. To achieve this, drugs have to be properly administered to the patient. In recent years many efforts have been made in the development of new drug delivery technologies (Bressan et al., 2009). Most of the difficulties to calculate the proper drug rate to each patient were the inexistence of precise methods to monitor the anaesthetic state of the patient. In the past, patient monitoring was performed just by observing several patient signs (sweat, head lifting, movement, etc.). Nowadays the way that anaesthesia is monitored has changed considerably.

Concerning hypnosis regulation, many efforts have been made to provide the anaesthesiologist with reliable methods for monitoring. In particular, the introduction of the Bispectral Index (BIS) to measure the depth of anaesthesia was one of the key elements in the development of new ways of drug administration (Sigl and Chamoun, 94).

The other main problem in designing control algorithms to regulate hypnosis arises from the complexity of the patient response to drug infusion. This response can be divided in two subsystems. One is the Pharmacokinetics (PK) that refers to the adsorption, distribution, biotransformation and excretion of the drug. And the other is the Pharmacodynamics (PD) that describes the equilibrium relationship between concentration in the body and visible effect produced in the patient. In practice a linear model has been accepted to describe the PK and a nonlinear model for the PD part. 
First works involved with anaesthesia control were focused in checking the performance of a fully automated controller compared with the results obtained in a process guided by an anaesthetist. In (Sakai et al., 2000) and (Morley et al., 2000) it is showed that proposed PID controller can assure intraoperative hemodynamic stability and a fast recover of the patient from the hypnosis effects of the drug using closed-loop techniques. In these works and in the works of Absalom (Absalom et al., 2002a, Absalom et al., 2002b, Absalom et al., 2003), it can be shown that the performance of the closed-loop system was as efficient as the observed in the process guided by the specialist, without demonstrating any clinical advantages over the manual techniques.

In the last decade, a lot of research related with automatic control of anaesthesia has been made. Most of them use the intravenous drug propofol as the hypnotic agent. It is important to mention the works that follow a signal-based control, as PID (Liu et al., 2006; Dumont et al., 2009) and fuzzy controllers (Gil, 2004), and the works that follow a model-based control. In this way, many different proposals have been made depending on the controller structure, the controlled variable and the prediction model used. In (Struys. et al., 2001; Sawaguchi et al., 2003; Furutani et al., 2005) the drug concentration in brain is used as controlled variable. In (Ionescu et al., 2008) and (Niño et al., 2009), were EPSAC tecniques are used as controller structure, in (Screenivas et al., 2008), were robust characteristics are added in the design of the controller, and in (S. Syafiie et al., 2009), were nonlinear techinques are used, the authors use predictive control techniques. In (Screenivas et al., 2009) a comparative study between predictive control and PID techniques applied to the control of anaesthetic is done.

The focus of this chapter will be in the regulation of depth of consciousness of patients under general anaesthesia with intravenous propofol.

\section{Anaesthetic process: The control problem}

The main variables that describe the anaesthetic process are depicted in figure 1 . In this figure an input-output description of the system is shown. As can be observed, manipulated variables are anaesthetics, relaxants or serums. Perturbations in the system are signals that can occur at any time (surgical stimulation, blood loss, etc.). The output variables can be measurable and not measurable. The main interest in anaesthesia is focused in nonmeasurable variables: hypnosis, analgesia and muscular relaxation. Although these variables are not directly measurable, there are methods to estimate them that are used in clinical practice. These methods are based on the use of alternative variables whose behaviour allows the estimation of the non-measurable ones.

Hypnosis is a general term indicating loss of consciousness and absence of the memory of the intervention after awake. Currently, the techniques that have been considered more efficient for this are based in the processing of the patient electroencephalogram (EEG), (Kazama et al., 1999; Struys et al., 2000).

The description of the BIS dynamics has been done mainly with physiological based models. These models consist of a PK part to describe the drug distribution in the internal organs and a PD part to describe the drug effect on the physiological variables of interest. 
The drug distribution in the body depends on transport and metabolic processes, which in many cases are not clearly understood. However, dynamical models based on conservation laws that capture the exchange of material between coupled macroscopic subsystems or compartments, are widely used to model these processes.

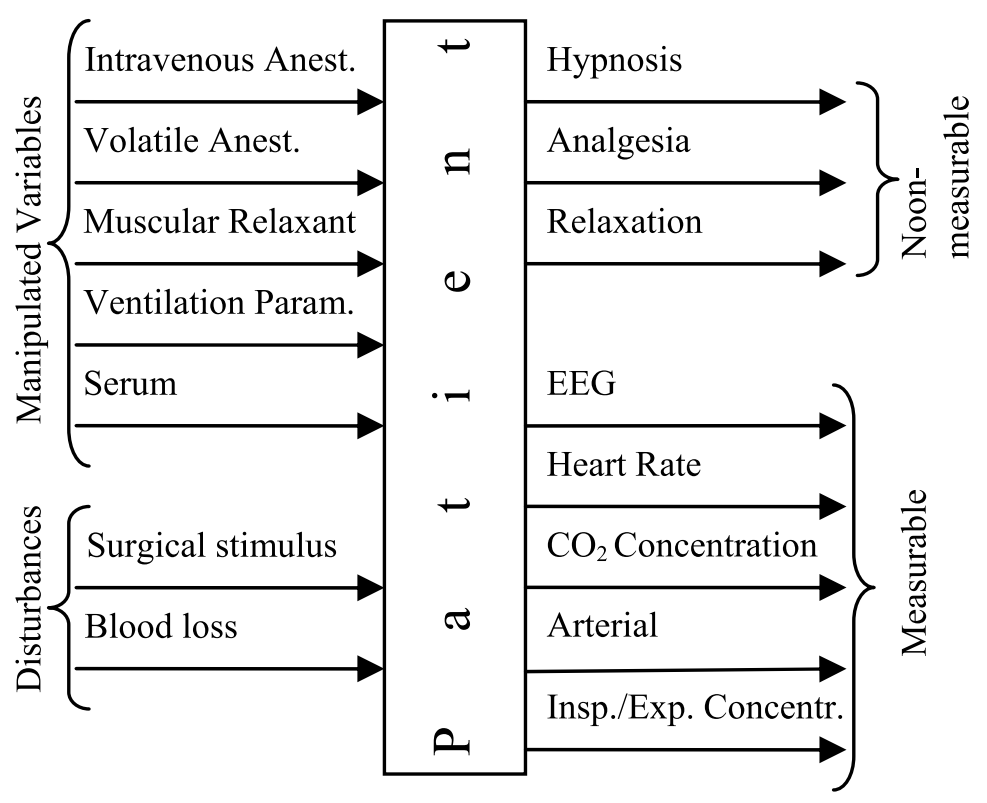

Fig. 1. Input-output description of the anaesthetic process.

\subsection{The compartmental model}

Figure 2 shows a model based in three compartments: central, fast and slow. The central compartment is the volume in which initial mixing of the drug occurs, and thus can be thought to include the vascular system (blood volume) and for some drugs the interstitial fluid. The fast peripheral compartment represents a compartment of the body that absorbs drug rapidly from the central compartment, and thus can be thought of as comprising tissues of the body that are well-perfused (such as muscles and vital organs). Finally the slow peripheral compartment is used to mathematically represent a compartment into which re-distribution occurs more slowly, and thus can be thought of as including tissues with a poor blood supply (such as adipose tissue). 


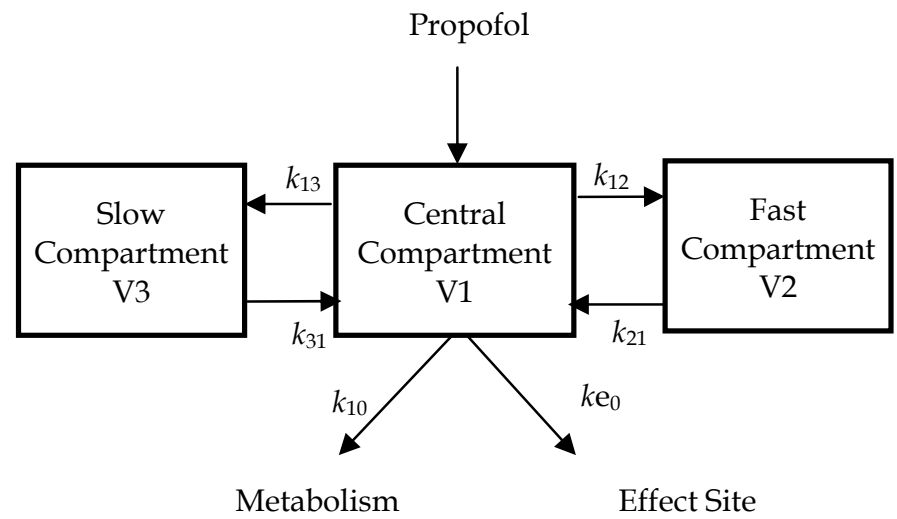

Fig. 2. Compartmental model.

The drug is infused in central compartment and then distributed to the slow and fast compartment and eliminated trough metabolism. Defining the drug concentration variable of the i-th compartment as $C_{i}$, the propofol distribution can be described as:

$$
\begin{gathered}
V_{1} \frac{\partial C_{1}}{\partial t}=V_{2} C_{2}(t) k_{21}+V_{3} C_{3}(t) k_{31}-V_{1} C_{1}(t)\left(k_{10}+k_{12}+k_{13}\right)+u(t) \\
V_{2} \frac{\partial C_{2}}{\partial t}=V_{1} C_{1}(t) k_{12}-V_{2} C_{2}(t) k_{21} \\
V_{3} \frac{\partial C_{3}}{\partial t}=V_{1} C_{1}(t) k_{13}-V_{3} C_{3}(t) k_{31} \\
\frac{\partial C_{e}}{\partial t}=C_{1}(t) k_{e 0}-C_{e}(t) k_{e 0}
\end{gathered}
$$

where $u(t)$ represents the drug infusion rate in the central compartment and $V_{i}$ is the volume of the i-th compartment. The dynamics of the compartmental model is defined by the following diffusion constants: $k_{10}$ (rate constant for drug metabolism), $k_{12}$ (rate constant for re-distribution of drug from central to fast peripheral compartment), $k_{21}$ (rate constant for redistribution of drug from fast to central compartment), $k_{13}$ (rate constant for redistribution of drug from central to slow compartment) and $k_{31}$ (rate constant for redistribution of drug from slow to central compartment). Common PK models for propofol are the Marsh model (Marsh et al., 1991) and the Schnider model (Schnider et al., 1998). Differences between both models can be seen in table 1 .

From the point of view of hypnosis control, the variable of interest is not the blood concentration but the concentration in the place where the effect on the controlled variable is produced (effect site concentration). Thus, when there is a simultaneous measure of the drug concentration in blood and its effect on the brain, drug latency can be observed that produces a temporal displacement between the peak of blood concentration and the drug effect. 
To include this dynamics in the model a fourth compartment is added. This compartment is known as effect site. It is assumed that this compartment is attached to the central compartment and has negligible volume. The diffusion constant of the effect site is $k_{e}$.

\begin{tabular}{|c|c|c|}
\hline & Marsh Model & Schnider Model \\
\hline$V_{\mathbf{1}}$ & $0.228 \mathrm{~L} / \mathrm{Kg}$ & $4.27 \mathrm{~L}$ \\
\hline$k_{10}\left(\mathbf{m i n}^{-1}\right)$ & 0.119 & $0.0443+0.0107^{*}(\mathrm{BW}-77)-0.0159^{*}(\mathrm{LBM}-$ \\
& & $59)+0.0062^{*}(\mathrm{HT}-177)$ \\
\hline $\boldsymbol{k}_{\mathbf{1 2}}\left(\mathbf{m i n}^{-1}\right)$ & 0.112 & $0.302-0.0056^{*}($ Age-53) \\
\hline $\boldsymbol{k}_{\mathbf{1 3}}\left(\mathbf{m i n}^{-1}\right)$ & 0.0419 & 0.196 \\
\hline $\boldsymbol{k}_{\mathbf{2 1}}\left(\mathbf{m i n}^{-1}\right)$ & 0.005 & $1.29-0.024^{*}($ Age-53 $)$ \\
\hline $\boldsymbol{k}_{\mathbf{3 1}}\left(\mathbf{m i n}^{-1}\right)$ & 0.0033 & 0.0035 \\
\hline $\boldsymbol{k e}_{\mathbf{0}}\left(\mathbf{m i n}^{-1}\right)$ & 1.21 & 0.456 \\
\hline
\end{tabular}

Table 1. Comparison of Marsh and Schnider models for PK of propofol. BW stands for Body Weight, LBM is Lean Body Mass and HT is Height.

On the other hand, the drug's pharmacodynamics, that represents the BIS in terms of the effect site concentration, is governed by:

$$
B I S=f\left(C_{e}\right)
$$

The $f$ function is usually taken as an EMAX model whose profile suits the described process:

$$
\begin{gathered}
\Delta B I S=\Delta B I S_{\max } \frac{C_{e}^{\gamma}}{C_{e}^{\gamma}+E C_{50}^{\gamma}} \\
\Delta B I S=B I S-B I S_{0} \\
\Delta B I S_{\max }=B I S_{\max }-B I S_{0}
\end{gathered}
$$

$\mathrm{BIS}_{0}$ corresponds to the awake state, $B I S_{\max }$ represents the minimum achievable BIS and $E C_{50}$ represents the concentration in the effect site for which the effect is half the maximum value, $\gamma$ represents the sensitivity of the patient to mall concentration variations in the effect site. This parameter can be seen as index that measures the degree of nonlinearity of the model.

\subsection{The control problem}

From the perspective of the control system three level of complexity can be distinguished. The basic procedure is the open-loop practice in which the anaesthetist, according to the parameters of the patient (age, weight, sex, ASA) directly uses predefined infusion rates of drugs. According to the response observed through his vital signs the drug rates can be modified (the anaesthetist is the controller).

In the next level, it appears the Target Controlled Infusion systems (TCI). In TCI the infusion rate is calculated from models of the pharmacokinetic of the patient, as can be seen in figure 
3. Thus, the objective in TCI is to achieve a pre-set target plasma concentration. According to the model of the patient the TCI system (normally implemented in the infusion pump) delivers the adequate drug doses to achieve the objective.

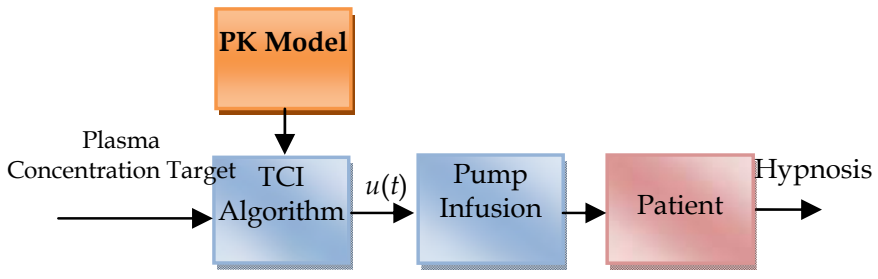

Fig. 3. Hypnosis control with TCI

There is a clear weakness in TCI related to the fact that the real plasma concentration cannot be online measured to compute the infusion rate. That is, TCI is also an open-loop control strategy. Closed-loop strategies appear to solve this problem. The main idea in closed-loop control is to use information of the state of the patient to automatically adjust the drug dosing. Many efforts have been made to provide the anaesthetist with more reliable methods for monitoring this state. In particular, the introduction of the Bispectral Index (BIS) to measure the depth of anaesthesia was one of the key elements in the development of new ways of drug administration (Sigl and Manchoun, 94). BIS has been demonstrated to correlate well with the depth of consciousness of the patient. Thus, it can be used as a feedback system to the controller in order to compute the adequate infusion rate, as can be seen in figure 4.

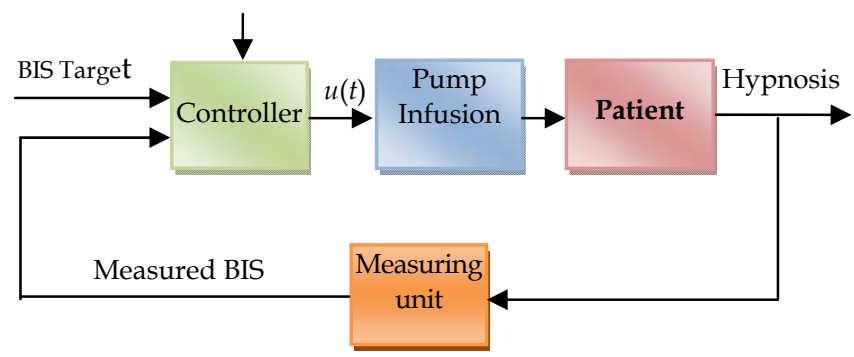

Fig. 4. Hypnosis control with closed-loop controller.

The controller algorithm used for anaesthesia control can be based on signals or in models. Signal based controllers are basically PID algorithms. The key feature of this algorithm is that no model is necessary to compute the infusion rate. Instead, the measured BIS is used to compute an error signal from which the drug dose is calculated. Model based controllers are an alternative to PID controller. The advantage of model-based controller is its ability to 
predict the behaviour of the patient and anticipate the changes in drug infusion to avoid undesirable responses.

In order to develop an adequate model-based control strategy, it is necessary to obtain a suitable model for the patient behaviour. One common practice is to simplify the model by means of linear approximations around a nominal state (corresponding to BIS target). On the other hand, models are also necessary for offline simulation of the controller structure. The most accepted models for patient representation are those based on compartments to represent the pharmacokinetic together with a nonlinear modelling that describes the PD.

\section{Implementation of the closed-loop control of anaesthesia}

The main elements that constitute the control system are depicted in figure 5 . As can be observed there is a computer that centralizes the monitoring and control task in the system. The BIS monitor is a passive analyser of EEG, that allows monitor the deep of anaesthesia, and has the first objective of adjust in real time the dose of drugs administered to one patient to the actual need. The BIS correlated well with the level of responsiveness and provided an excellent prediction of the level of sedation and loss of consciousness for propofol and midazolam. In this work the Aspect ${ }^{\circledR}$ A-2000 monitor was used. The communication with the computer was implemented via a RS-232 serial interface. Concerning the actuator, the Graseby ${ }^{\circledR}$ infusion pump was used for drug infusion in the patient. The pump is also governed via a RS-232 serial interface.

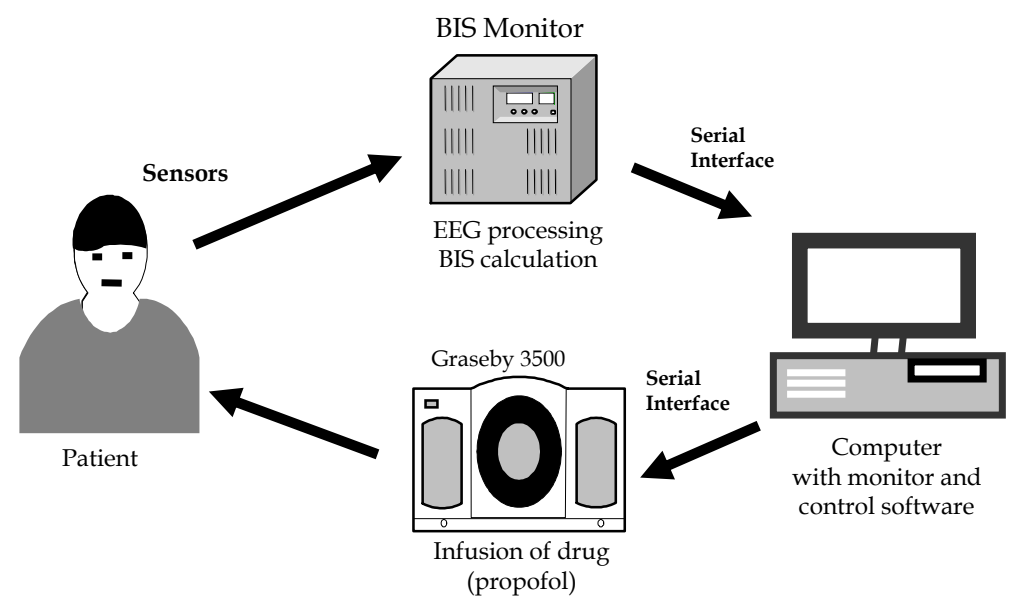

Fig. 5. Main elements of the closed-loop control system.

Apart from sending commands to the pump, the program in the PC reads continuously its state to detect eventual failures of any of the elements in the control loop, like missing BIS 
signal, excessive infusion rate in the pump syringe changes, etc. The program in the computer has all the routines to monitor and control the system.

The goal is to make a manual induction with propofol and remifentanil and maintain the BIS target during the maintenance of anaesthesia. Remifentanil infusion was adjusted manually and rocuronium was administered in bolus as needs. The operation conditions and the population in which the study was performed are explained in next section.

\section{PI control}

First control algorithm implemented was a PI controller. This algorithm has been extensively used in several automated closed loop systems. The administration of the drug is made based in the error between the BIS target and the current BIS, and the accumulated error during the operation. Both actions are regulated by gains, which are adjusted in an empirical way trying to get a smooth transitory and a stable response of the patient.

The goal is to make a manual induction with propofol and remifentanil and maintain a BIS target during the maintenance of anaesthesia. Reminfentanil infusion was adjusted manually and rocuronium was administered in bolus as needs. In the real proofs, a BIS target $\left(\mathrm{BIS}_{\mathrm{r}}\right)$ of 50 is considered while the measurement and actuation period is 5 seconds. Before starting its operation, the software checks that all the security alarms are programmed.

This study was approved by the Ethical and Research Committee of the Hospital Universitario de Canarias and has written informed consent of the patients. The study was performed on a population of 15 patients of 30-60 years. In the real proofs with patients, a BIS target of 50 is considered while the measurement and actuation period is 5 seconds. Before starting its operation, the software checks that all the security alarms are programmed. In the operating room, the patient was connected to the BIS monitor, and the anaesthesia system was started in monitor mode. After the patient had breathed $100 \%$ oxygen for $3 \mathrm{~min}$, the system was switched to manual mode, and anaesthesia was induced by means of nearly $2 \mathrm{mg} / \mathrm{Kg}$ propofol manual bolus. Once the patient achieves a BIS closed to 50 , the system is switched to automatic and the interest control algorithm is responsible to regulate the BIS around the objective.

The adjustment of the controller gains was made in an empirical way trying to get a smooth transitory and a stable response. This task was done following standard procedures in online process control engineering. For this, it was necessary the presence of a control expert together with the anaesthesiologist in the operating theatre. Thus, after several trials adequate values for PI controller where found to be $K p=0.67, K i=0.055$. This set of values was tested in the whole population of the study with satisfactory results. Figures 6 and 7 present the evolution of the anaesthesia for two different patients: patient 1 and patient 2 . As can be observed, in both cases the system remains stabilised around the reference value with an oscillation of near \pm 10 units in the worst case (patient 2).

The results obtained with the population submitted to proofs show the patient remains stabilised around the reference value with an oscillation of near \pm 10 units in the worst case. 

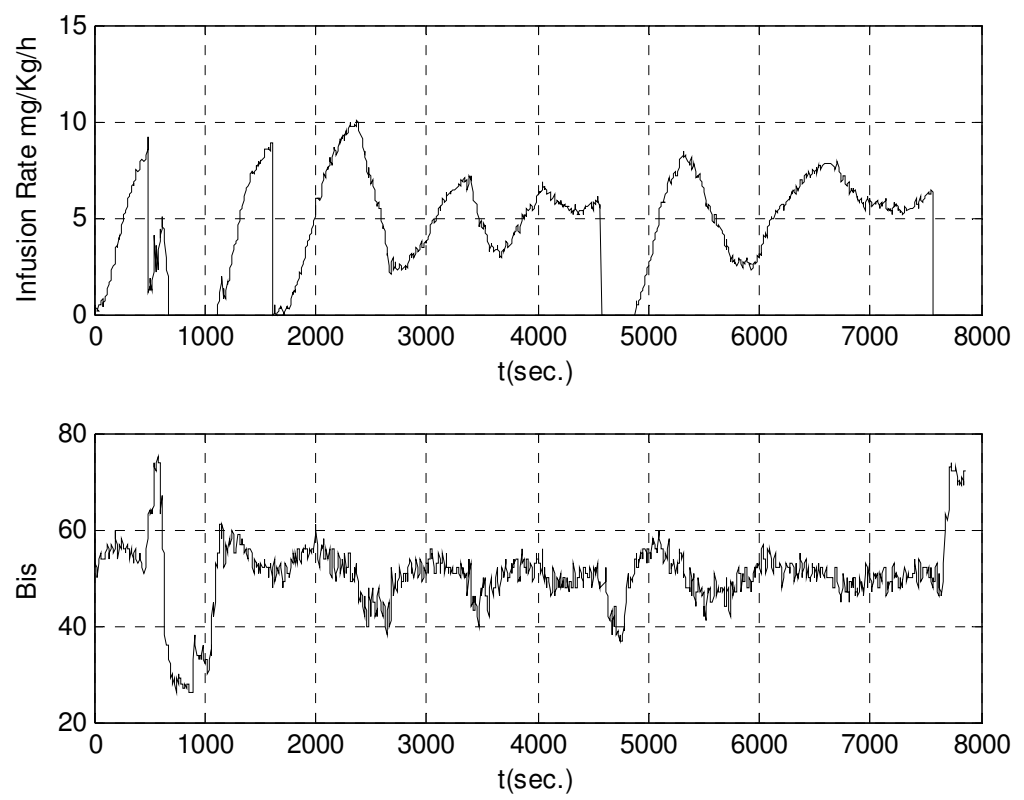

Fig. 6. Results of anaesthesia automatic control on patient 1.
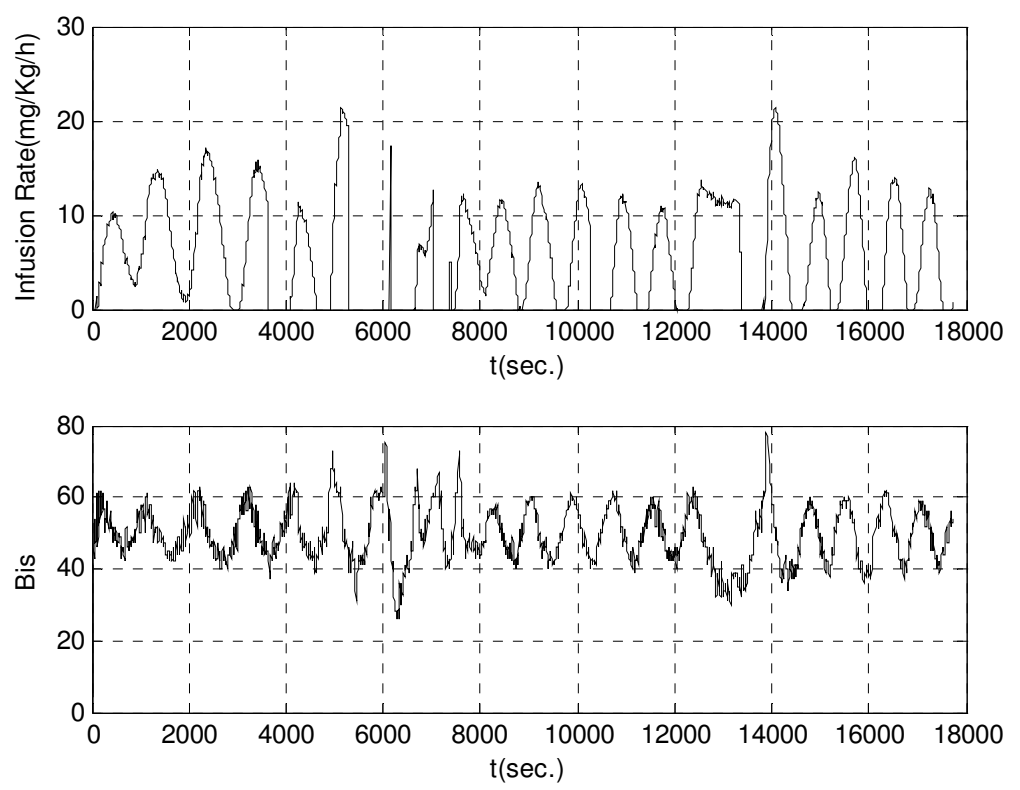

Fig. 7. Results of anaesthesia automatic control on patient 2. 
The study revealed that although results are satisfactory, eventually the performance of the controller could decrease. There are two main factors that contribute to this. First of all, the variability between patients implies that the nominal PI parameters chosen are not the best choice for all the patients. Together with this, the dead time present in the system also contributes to reduce the phase margin of the closed loop system. The origin of this timedelay is the period of time since the drug is infused until it causes the adequate effect in the patient. The main effect observed is that the evolution of the BIS is quite oscillatory (see figure 7) around the reference value.

\section{Dead-time compensation}

In previous section it can be viewed that PI controller usually gives a response with oscillations around the BIS reference value. In this section, the control algorithm is modified in order to compensate these oscillations and get a better transitory. The results shown in this paper are in simulation after having adjusted the patient dynamical model.

The first method implemented to improve the results obtained with the fixed PI controller is to compensate the dead-time present in the system. To do this, a dead-time compensator based on the Smith Predictor theory (Smith, 72) is proposed to act with the PI controller. The basis of the Smith Predictor is to consider the feedback of the controlled variable BIS without delay. As this is variable is not available, the predictor estimates this value and uses this estimation as the feedback signal. To correct the deviations between this estimation and the real value, a correction term, resulting from the error between the estimation and the measured BIS, is added to the feedback signal, as can be seen in the figure 8 .

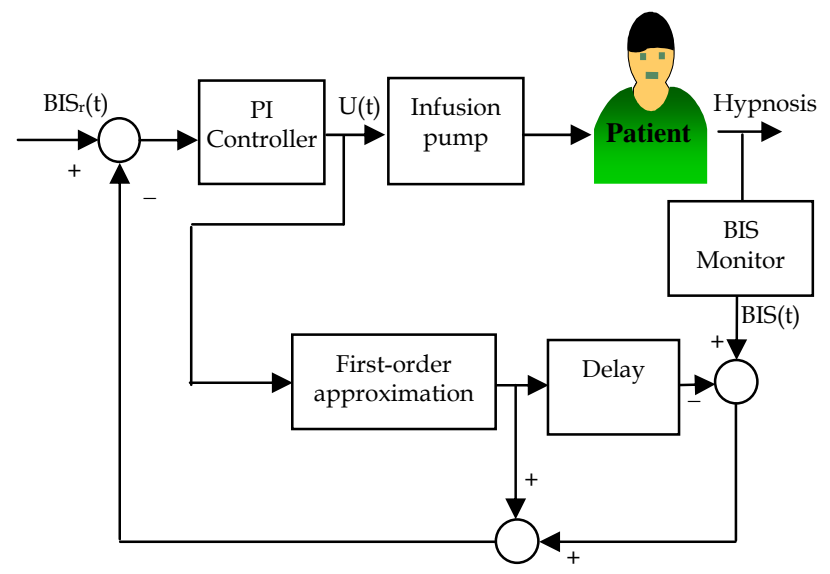

Fig. 8. PI Controller with Smith Predictor for patient hypnosis control.

The basics of this compensation algorithm consider the formulation of the Smith Predictor for linear systems. To apply the Smith Predictor to the nonlinear model of the patient, a firstorder plus a time-delay approximation of the patient model is considered. A delay between 90 and 120 seconds is considered. 


\subsection{Model adjustment}

In order to make the simulations proofs of the proposed algorithm, a physiology model of the patient dynamics was designed. As it was told, the model has two parts: pharmacokinetics and pharmacodynamics. The parameters adjustment was made in simulation using the real results obtained from a female, 56 years old patient, $84 \mathrm{Kg}$. weight, $160 \mathrm{~cm}$. height.

After obtaining a satisfactory manual adjustment, the values for the pharmacokinetics model are $\mathrm{k} 10=0.006, \mathrm{k} 12=11.0 ; \mathrm{k} 21=14.04, \mathrm{k} 13=10.02, \mathrm{k} 31=283.50$ and $\mathrm{ke} 0=0.0063$. The values for the pharmacodynamics model are EC50=610.0, $\gamma=1.5, \mathrm{BIS}_{0}=100$ and $\mathrm{BIS}_{\max }=0$.

To validate the model the simulated response is compared with the real one. The obtained results, shown in figure 9a), prove the goodness of the model.
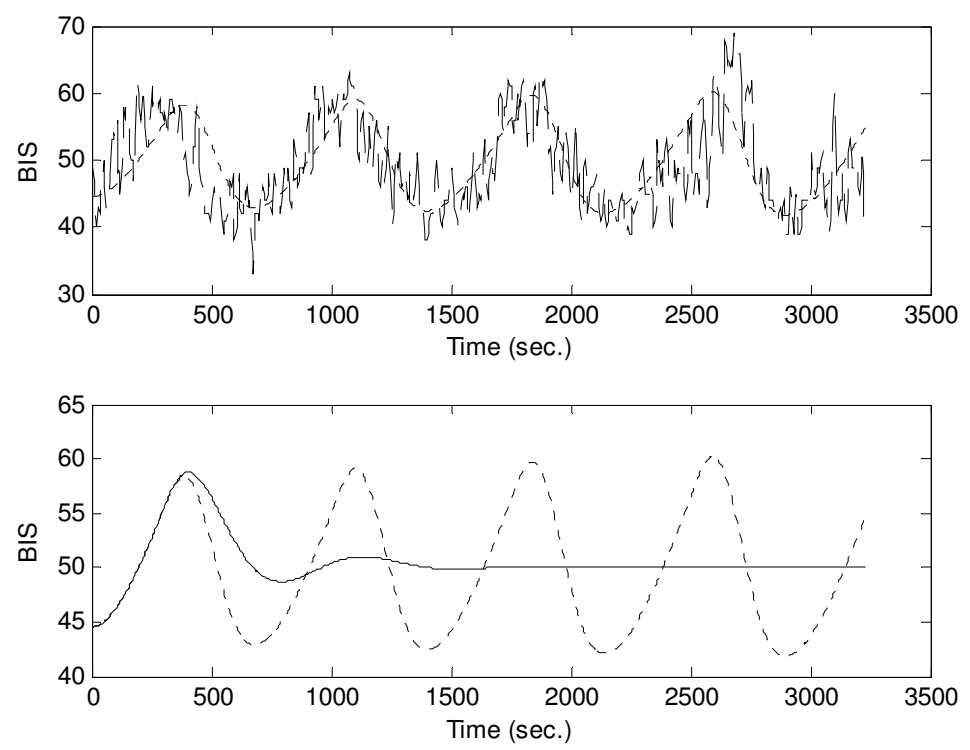

Fig. 9. a) Simulated BIS output (dotted) and real patient BIS output (solid) obtained under the action of a PI controller. b) PI controlled output (dotted) and PI with delay-time compensation output (solid).

\subsection{Smith predictor (dead-time compensation)}

The proposal here is to improve the performance of the closed-loop system by means of a compensation of the system time-delay. The origin of this time-delay is the period of time between from the infusion pump starts until the drug is distributed along the central compartment. The majority of the works in the literature do not explicitly consider the presence of this time-delay in the proposed models. In fact, in model equations (1)-(4), timedelay is not considered. But in real proofs, some delays between 1 and 2 minutes have to be considered to have a realistic model of the dynamics. Under this hypothesis, a time-delay 
compensator based on the Smith Predictor theory has been proposed to be added to the PI controller.

\subsection{Results with PI controller with dead-time compensation}

As it is well known, the basics of this compensation algorithm consider the formulation of the Smith Predictor for linear systems. To apply the Smith Predictor to the nonlinear model of the patient, a first-order plus a time-delay approximation of the patient model is considered. Thus, the configuration employed can be seen in figure 8 . A delay between 90 and 120 seconds is considered in the simulations. In figure $9 \mathrm{~b}$ the results obtained with the patient are shown. The evolution of the BIS signal with the Smith Predictor (in solid line) is much better than with the PI controller (in dotted line), and does not show oscillations around the reference BIS value.

\subsection{Self-adaptive dead-time compensation}

The main advantage of the time-delay compensation for the PI controller is a better performance in the transitory of the BIS signal. This advantage is conditioned to obtain a good fist-order approximation of the system. However, this model has to be changed in at least two situations. First, when the operation point changes due to a change in the BIS reference for the same patient. Second, when the controller is applied in a different patient, whose physiologic model has to be estimated.

In order to improve the efficiency of that controller, an adaptation of the first-order model patient is added. The aim of this algorithm is to make the time-delay compensator independent of the model assumed for the patient. In order to obtain a simple adaptive algorithm that guarantees the closed-loop stability, model reference adaptive controller MRAC- (Aström and Wittenmark, 94) is used, as can be seen in figure 10.

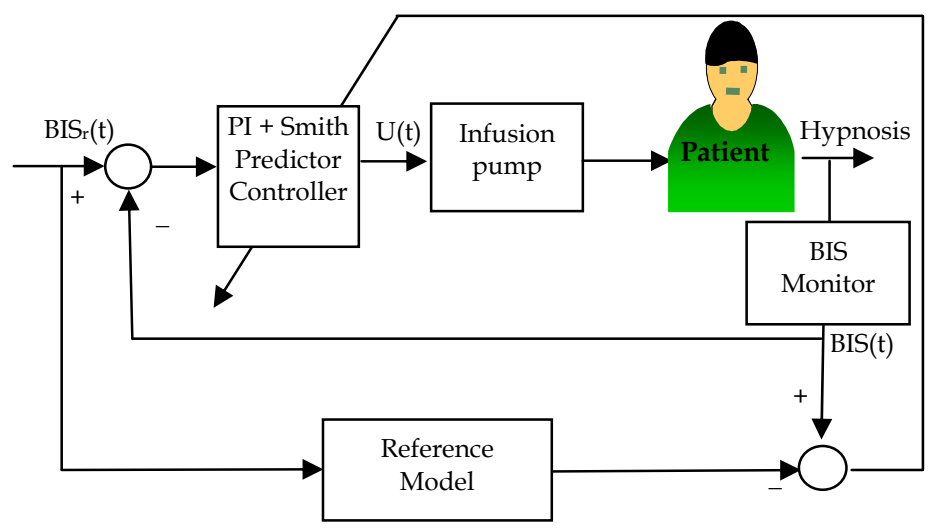

Fig. 10. PI with Smith Predictor controller inserted in the adaptive control scheme MRAC. The error between the system output and the model reference output is used to update the parameters of the PI with Smith Predictor controller. 
Following this control scheme, the controller parameters are adjusted by an adaptation law that depends on the error between the system output (BIS) and the model reference output defined for this closed-loop. Minimising a certain cost-function involving this error, an adaptation law of the adjustable controller parameters is obtained. In this case, the adjustable parameters are the static gain and the time constant of the approximated firstorder model used in the Smith Predictor.

\subsection{Results with the PI controller with self-adaptive dead-time compensation}

Several simulation experiments has been made for the patient simulated in previous sections, choosing as the reference model a second-order model with poles, expressed in the $\mathrm{z}$-plane discrete formulation, in $\mathrm{z}=0.98$ and $\mathrm{z}=-0.75$.

The results are shown in figure 11a), where the evolution of the BIS under the self-adaptive compensator algorithm is drawn in solid line and compared with the results obtained in figure 9b) -PI and PI+compensator controllers-. In figure 11b) the evolution of the static gain under this self-adaptive scheme is shown. As it can be observed, some extra oscillations are produced with respect to the previous algorithm, which corresponds to the period of time in that the parameter is adapting.
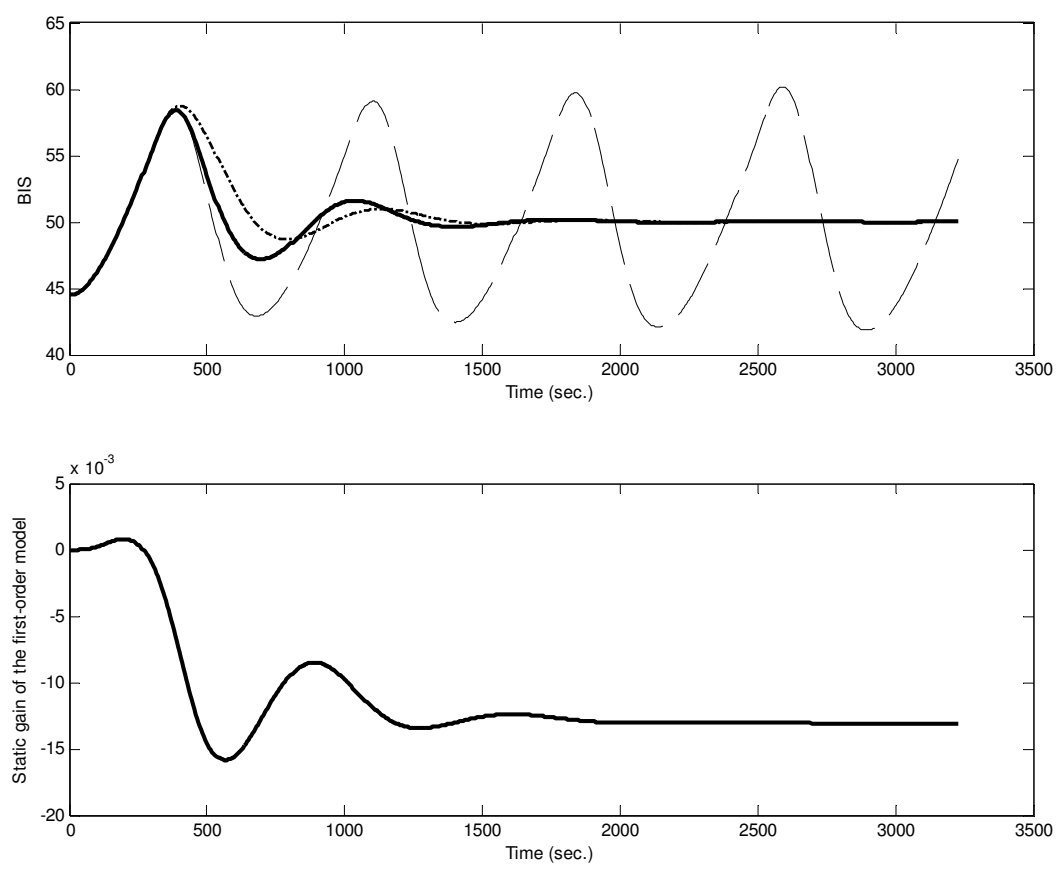

Fig. 11. a) PI controlled system output (dotted), PI with Smith Predictor controlled output (dashed), and self-adaptive time-delay compensation controlled output (solid) compared for the same patient. b) Evolution of the static gain of the first-order approximation model of the time-delay compensation. 
Once the optimal values for the parameters are reached, the performance of the system is very similar to the previous controller. In that case, the static gain took a value of -0.037 . In this case, the stabilising value for this parameter is near the half. However, the performance is also satisfactory. Moreover, no assumptions over the system had to been made, which is the main advantage of this new algorithm.

Comparing the results obtained with the previous algorithm, some extra oscillations are produced, which corresponds to the period of time in that the parameter is adapting. Once the optimal values for the parameters are reached, the performance of the system is very similar to the previous controller. Moreover, no assumptions over the system had to been made, which is the main advantage of this control algorithm.

\section{PI control with self-adaptive gains}

Another method to avoid the problem of the oscillations around the BIS reference value occurred with the PI controller is using an adaptive scheme to obtain the gains of the PI controller. The method is based on assuring a desired performance of the closed loop, by emplacing its poles as required, in order to obtain a smooth transition to the BIS reference value.

This method is part of a kind of adaptive controllers, known as self-tuning regulators -STR(Aström and Wittenmark, 94). They are based on two steps. First, an identification of the system is made in order to get the dynamics in each instant of time. This assures that the controller takes into account the variations in the dynamics of the system. Second step is to compute the control law assuming that the identification results are true. The variety of STR schemes differs in the method used to compute the control law. In this work, an adjustable PI controller is used, which gains are tuned by trying that the closed loop performance of the system be as similar as possible to a desired reference model. This is obtained by emplacing the poles of the reference model into the desired values to get a satisfactory response of the system.

STR controllers have two different parts. The most important part is that they provide an observer in order to identify the dynamics of the system to control. This is an essential point due to the results of the system identification are taken into account to compute the adaptive control law. In this case, a recursive least-squares method based is used to provide the parameters of the dynamics of the observed system.

The second part of the controller is to compute the control law to apply to the system. The results of the identification part are taken to obtain the control action. In this way, the controller takes into account the variations of the dynamics of the system, if they occur. Another advantage of this scheme is its flexibility to be applied to different systems, with the same dynamics but with different parameters, because the identification process adapts the controller to the new situation.

In this case, a pole placement controller is used. Considering the dynamics of the patient as a first order system, and using a PI controller with adaptive gains, the closed loop performance results in a second order system, which dynamics are set by emplacing its poles to the desired values for the closed loop system. Next subsections describe these two parts of the proposed controller. 


\subsection{Patient dynamics identification}

To obtain the dynamics of the patient, a stochastic recursive least-squares method is employed. It consists of an observer of the discrete system that tries to minimize the following cost function:

$$
J=\frac{1}{2} \varepsilon^{2}(k)
$$

where $\varepsilon(k)$ is the residual error between the output of the system, BIS $(k)$, and the observed model, being $k$ the instant of time.

Consider the following first order model as an approximation of the patient dynamics, with $u(k)$ being the drug infusion rate of applied to the patient, and BIS $(k)$ the BIS value obtained:

$$
B I S(k)+a_{1} B I S(k-1)=b_{1} u(k-1)
$$

It can be expressed by:

$$
\begin{gathered}
y(k)=\phi^{T}(k) \theta \\
\varphi(k)=[-B I S(k-1) \quad u(k-1)]^{T} \\
\theta=\left[\begin{array}{ll}
a_{1} & b_{1}
\end{array}\right]^{T}
\end{gathered}
$$

In each instant of time, the values of output and input of the system are measured. The iterative process to obtain the best parameters $\theta$ that reproduce the performance of the patient is given by the equations:

$$
\begin{gathered}
e(k)=y(k)-\phi^{T}(k) \theta(k-1) \\
\theta(k)=\theta(k-1)+W(k) \phi(k) e(k)
\end{gathered}
$$

where:

$$
W(k)=\frac{c}{k}
$$

being $c$ a constant.

\subsection{Pole placement controller}

The control law considered for the system is obtained by applying a PI controller. This controller has two gains to adjust: the proportional gain, $\mathrm{Kp}$, and the integer gain, Ki. The discrete version of this controller is given by:

$$
G_{c}(k)=\frac{\alpha+\beta z^{-1}}{1-z^{-1}}
$$


where the relation with the gains is given by:

$$
\begin{gathered}
\alpha=K_{p}+K_{i} \frac{T}{2} \\
\beta=-K_{p}+K_{i} \frac{T}{2}
\end{gathered}
$$

being $T$ the sampling time of the system. Considering for the patient the model (10) and for the controller the expression (17), the poles of the closed loop are given by the roots of the following polynomial:

$$
D\left(z^{-1}\right)=\left(1+a_{1} z^{-1}\right)\left(1-z^{-1}\right)+b_{1} z^{-1}\left(\alpha+\beta z^{-1}\right)
$$

Consider the following specification for the closed-loop:

$$
Q\left(z^{-1}\right)=\left(1-q_{1} z^{-1}\right)\left(1-q_{2} z^{-1}\right)
$$

where $q_{1}$ and $q_{2}$ are the location of the desired poles for the system. It is easy to obtain from (20) and (21) the resulted gains of the PI controller:

$$
\begin{gathered}
\alpha=\frac{-1}{b_{1}}\left(1+q_{1}+q_{2}+a_{1}\right) \\
\beta=\frac{1}{b_{1}}\left(q_{1} q_{2}+a_{1}\right)
\end{gathered}
$$

The parameters of the system are obtained in each instant of time form the identification process (15). With the values (22) and (23), the gains of the PI controller are obtained, using (18) and (19), by:

$$
\begin{aligned}
& K_{p}=\frac{\alpha-\beta}{2} \\
& K_{i}=\frac{\alpha+\beta}{T}
\end{aligned}
$$

\subsection{Results with the PI self-adaptive controller}

The results shown here correspond to a female of 40 years old, $70 \mathrm{Kg}$. weight and $170 \mathrm{~cm}$ height. The Schnider model presented in section 2 gives an accurate response with the real values obtained for the patient, where the following parameters for (6)-(8) equations are chosen: $\mathrm{BIS}_{0}=95, \mathrm{BIS}_{\max }=8.9, \mathrm{EC}_{50}=4.94 \mu \mathrm{g} / \mathrm{ml}$ and $\gamma=2.69$. Initially, the patient is infused with a bolus of $1.45 \mathrm{mg} / \mathrm{Kg}$ during 2 minutes in order to carry the patient near the desired value for the degree of hypnosis. The reference value is $\mathrm{BIS}_{\mathrm{r}}=50$. After that, the compensated adaptive controller starts controlling the system.

To define the controller, the following assumptions are considered. First, the model for the patient is chosen as: 


$$
B I S(k)-0.812 B I S(k-1)=-25.389 u(k-1)
$$

For the second-order reference model, the poles are located in $0.997 \pm 0.0027 \mathrm{j}$ that correspond to a system with natural frequency $0.05 \mathrm{rad} / \mathrm{sec}$ and delta coefficient 0.75 . For initiating the identification algorithm (15), the parameters used in (26) are chosen. $c$ constant in (16) is set to 0.005 .

Figure 12 shows the evolution of the BIS and the parameters of the PI controller in this case. As it can be seen, after the application of the initial bolus, the PI controller varies its gains and the patient remains its degree of freedom around the desired value of 50 , but with oscillations.
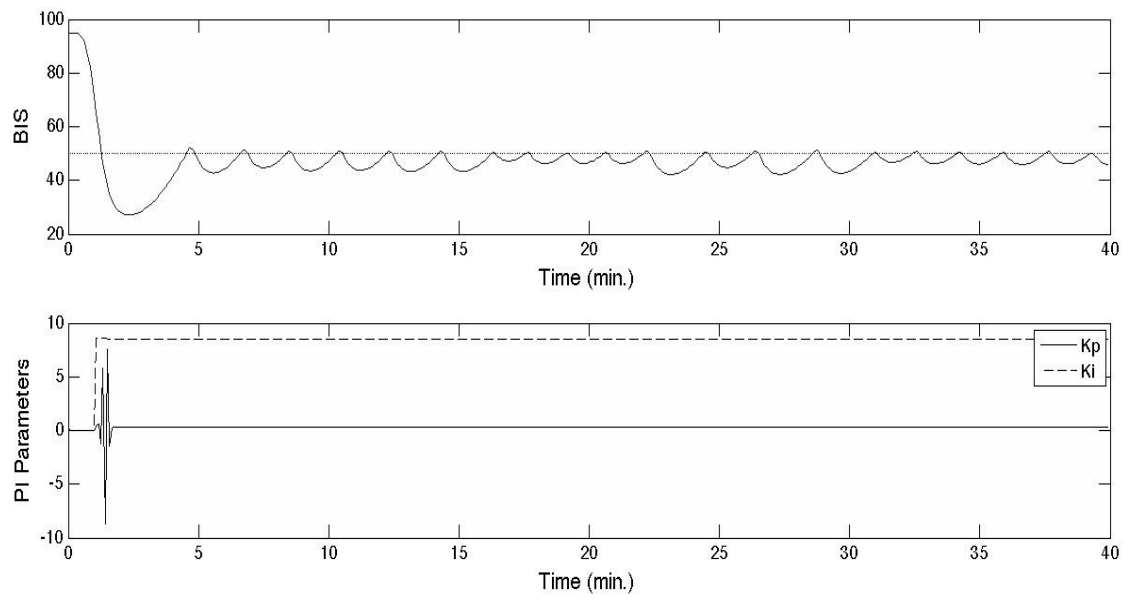

Fig. 12. Results of the PI self-adaptive controller. First graph shows the BIS evolution with respect to $\mathrm{BIS}_{\mathrm{r}}$ and second graph are the gains evolution of the PI controller.
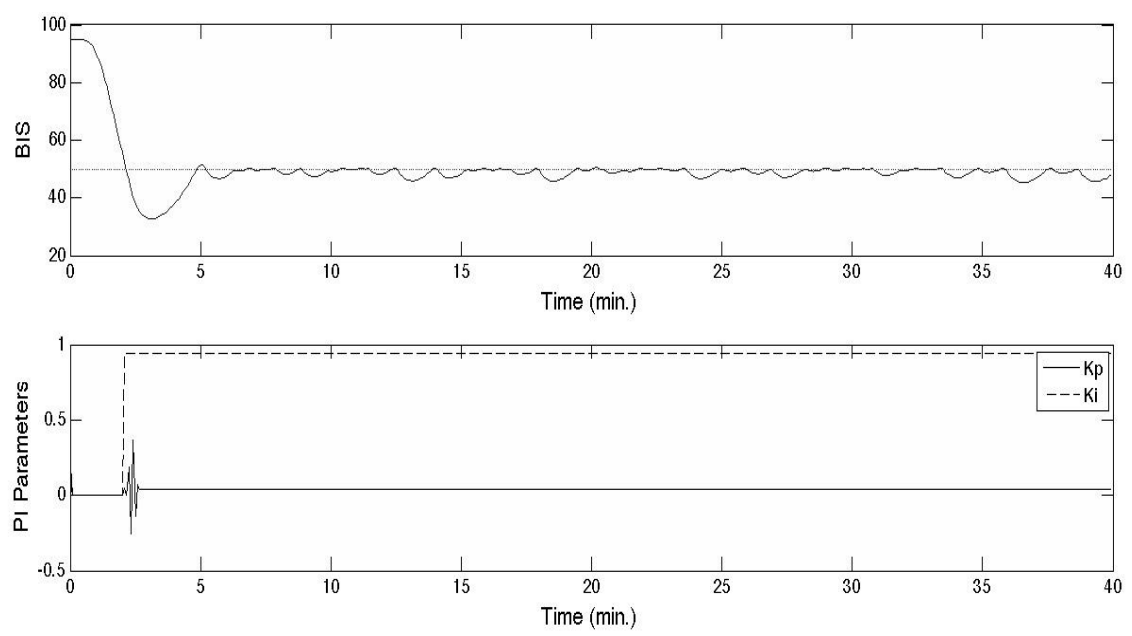

Fig. 13. Results of the compensated self-adaptive PI controller. First graph shows the BIS evolution with respect to $\mathrm{BIS}_{\mathrm{r}}$ and second graph are the gains evolution of the PI controller. 
To avoid these oscillations in the stationary, the compensation of the time delay by means of a Smith predictor is done in this case. To do that, the time delay considered is 1 minute. Figure 13 shows the results obtained in this case. The oscillations around the objective value are considerably reduced and the performance of the closed loop systems results better than in previous case.

\section{Model Predictive Control (MPC)}

As an alternative to signal based controllers proposed before, it is shown an algorithm that uses explicitly the model of the patient to compute the drug infusion rate. The objective is to improve the performance of other techniques as those based in PI controllers. Figure 14 shows the structure of the proposed controller. As can be observed, the drug infusion rate is computed as a sum of two terms:

$$
u(t)=u_{n}+\delta u
$$

The first term $\left(u_{n}\right)$ is obtained by inverting the model of the patient and is computed to take the BIS variable to the nominal value $\left(\mathrm{BIS}_{r}=50, x_{\mathrm{n}}\right)$. That is, from the target BIS $\left(\mathrm{BIS}_{r}\right)$ and using the model of the patient (PK+PD) it can be obtained the infusion rate that leads the BIS signal to the desired value. To do this, the inverse dynamics of the system model is evaluated, assuming that the system is approximated by (1)-(5). Taking matrix notation for this model, it can be expressed by:

$$
\dot{x}=A x+B u
$$

where $x=\left[\begin{array}{llll}C_{1} & C_{2} & C_{3} & C_{e}\end{array}\right]^{T}$.

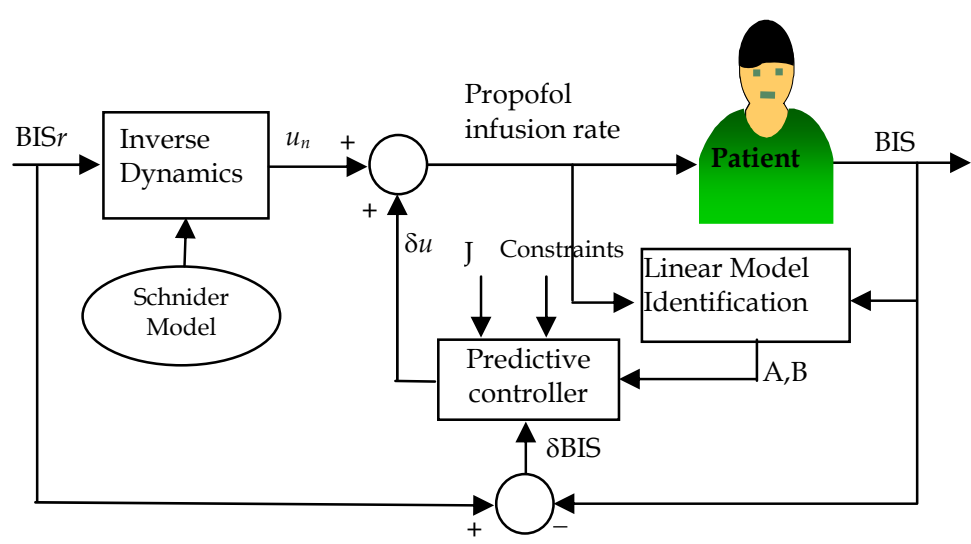

Fig. 14. Structure of the proposed model-based predictive controller. 
Assuming that the target state is an equilibrium state $x_{n}=\left[\begin{array}{llll}C_{1 n} & C_{2 n} & C_{3 n} & C_{e n}\end{array}\right]^{T}$, the nominal input can be obtained by solving:

$$
A x_{n}+B u_{n}=0
$$

$C_{e n}$ is computed by using the EMAX model (6), (7) and (8):

$$
C_{e n}=\exp \left(\frac{1}{\gamma} \ln \left(E C_{50}^{\gamma} \frac{\alpha}{1-\alpha}\right)\right)
$$

with:

$$
\alpha=\frac{B I S_{r}-B I S_{0}}{B I S_{\max }-B I S_{0}}
$$

Finally, to compute the nominal input $u_{n}$ and the nominal state $x_{n}$ equation (29) is solved to obtain the following solution:

$$
x_{n}^{\prime}=M^{-1} N
$$

where $x_{n}^{\prime}=\left[\begin{array}{llll}C_{1 n} & C_{2 n} & C_{3 n} & u_{n}\end{array}\right]^{T}$ and:

$$
\begin{gathered}
M=\left[\begin{array}{llll}
A_{11} & A_{12} & A_{13} & B_{1} \\
A_{21} & A_{12} & A_{23} & B_{2} \\
A_{31} & A_{32} & A_{33} & B_{3} \\
A_{41} & A_{42} & A_{43} & B_{4}
\end{array}\right] \\
N=-\left[\begin{array}{l}
A_{14} C_{e n} \\
A_{24} C_{e n} \\
A_{34} C_{e n} \\
A_{44} C_{e n}
\end{array}\right]
\end{gathered}
$$

In Fig. 15, a simulation of the BIS in a patient with only this nominal input $\left(u_{n}\right)$ is presented.

As can be observed, the BIS tends to the nominal value $\left(\mathrm{BIS}_{\mathrm{r}}=50\right)$ if only this input is applied. In practice, several considerations have to be taken into account. The first one is related to the modeling errors in the patient dynamics. In the simulation presented here no modeling errors were considered. In the real implementation, a deviation of the response of the system with respect to this ideal trajectory will be observed. On the other hand, as can be observed, the response exhibits a sluggish behavior that in real practice is undesirable. That is why this action is complemented with an additional term that tries to correct the deviations of the system from the nominal trajectory and also improves the transient response of the BIS curve. 


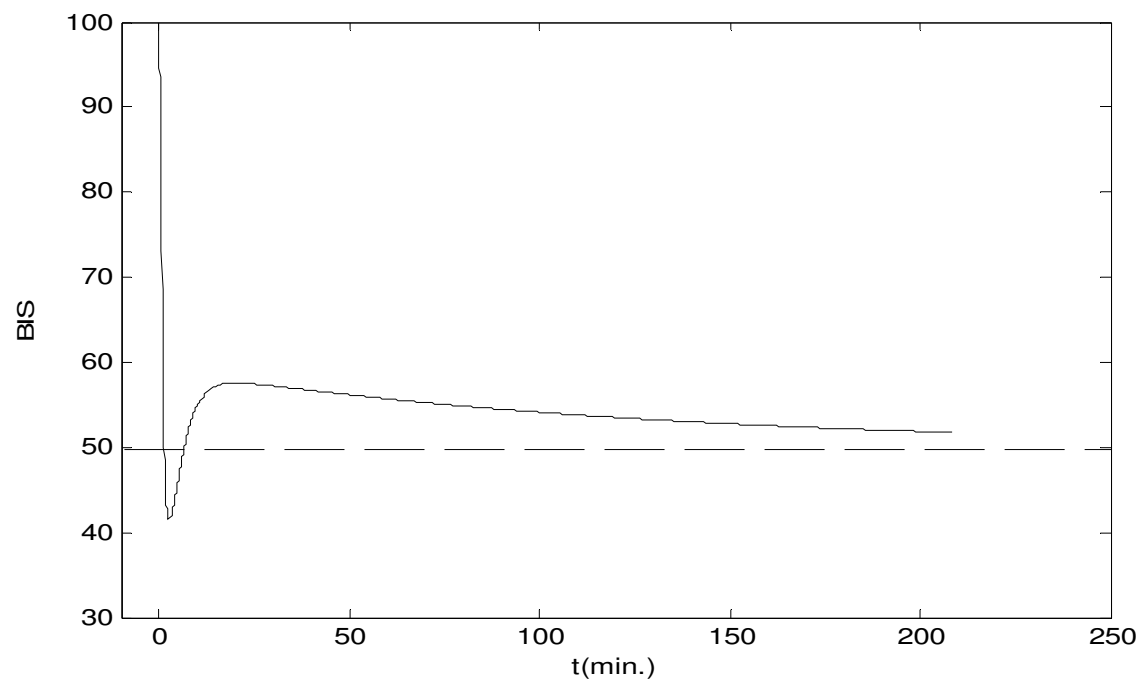

Fig. 15. Simulation of the BIS in a patient applying only the nominal input $u_{n}$.

This action is computed considering that the deviations of the BIS from the nominal value can be described by a linear approximation:

$$
\begin{gathered}
\delta \dot{x}=A \delta x+B \delta u \\
\delta B I S=C \delta x+D \delta u
\end{gathered}
$$

where $\delta x=x-x_{n}$ and $\delta B I S=B I S-B I S_{r}$.

Then the control law $\delta u_{k}$ can be obtained from an optimization problem (model based predictive controller). The problem can be formulated as obtaining the control law $\delta u_{k}$ so that a specified cost function is minimized under a receding horizon strategy. Consider for example the following index:

$$
J_{k}=\sum_{j=1_{1}}^{N} \gamma(j)[w(k+j)-\hat{y}(k+j \mid t)]^{2}+\sum_{j=1}^{N U} \lambda(j)[\delta u(k+j-1)]^{2}
$$

where $N$ is the prediction horizon and $N U$ is the control horizon. The problem is to find the sequence $\delta u_{k+j}$ so that $J_{k}$ is minimized. Assuming a receding horizon strategy, only the first value of the sequence is applied and the procedure is repeated at $k+1$. In this optimization, constraints can also be included, although the computational complexity is greatly increased.

Figure 16 shows a simulation of this strategy. Initial condition was BIS=39. As can be observed, the response of the system is now much faster than that observed in figure 14 and achieves a very acceptable error quite soon. 

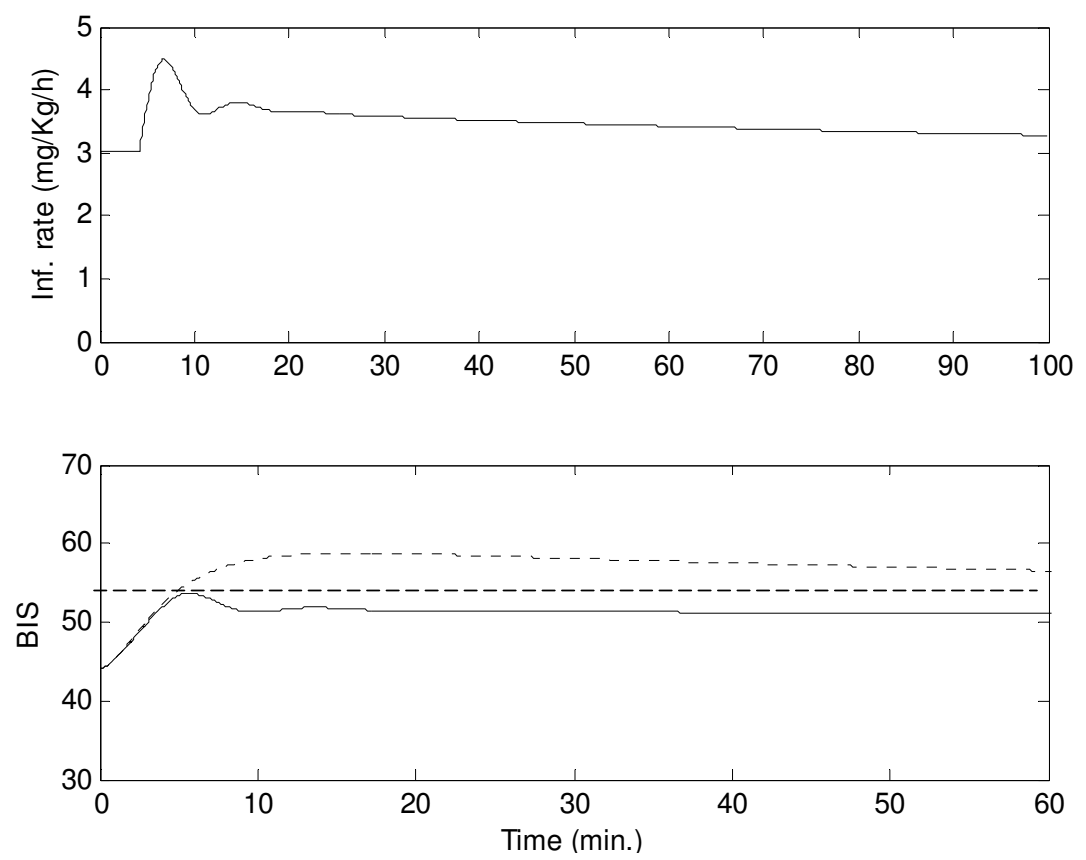

Fig. 16. Simulation of the model-based controller of figure 14 on a patient. Nominal input (dashed line) and nominal+predictive controller (solid line).

\section{Conclusion}

In this chapter both results on modelling and control of patients under general anaesthesia with propofol is presented. First results presented refer to the synthesis of linear models for use in model-based controllers. The anaesthetic process was segmented into several phases, according to the state of the surgery (consciousness, hypnosis, intubation, incision, etc.). The propofol infusion rate in $\mathrm{ml} / \mathrm{h}$ was used as the input variable $u(t)$, while the BIS represented the output. Validation of the proposed model was done with real data patients.

For simulation purposes a PK/PD model based on compartmental approaches as obtained. The model was adjusted using information of real data from patients. The obtained model was used to simulate the response of the patients with the different controllers.

Concerning hypnosis control, this chapter presents a review of the state of the art of the closed-loop control of anaesthesia. Then, a description of approaches based on signal feedback and model based controllers are presented.

The chapter proposed an advanced PI controller with several important features. First, an adaptive module is included that adapts the controller to the specific patient behaviour. On the other hand, the controller incorporates a dead-time compensation system that improves notably the performance of the controller. The performance of this compensation is 
improved by self-adapting the approximation of the patient model used in the Smith Predictor scheme.

The last part of the chapter is devoted to the design of model-based controllers. In particular, a model-based predictive controller is presented that corrects efficiently the transient evolution of the BIS, offering a smooth evolution of this signal to the reference value.

\section{Acknowledgment}

This work is under the auspicious of the Research Project DPI2010-18278 supported by "Ministerio de Educación y Ciencia" of the Spanish Government.

\section{References}

Absalom, A.R., Sutcliffe, Kenny, G.N.C., (2002a). Closed-loop Control of Anesthesia Using Bispectral Index. Performance Assessment in Patients Undergoing Major Orthopedic Surgery under Combined General and Regional Anesthesia. Anesthesiology; 96. pp.6773.

Absalom, A.R.; Leslie, K.; Kenny, G.N.C. (2002b). Closed loop control of sedation for colonoscopy using the Bispectral Index. Anaesthesia, 57. pp. 690-709.

Absalom, A.R.; Kenny, G.N.C. (2003). Closed-loop control of propofol anaesthesia using bispectral indexTM: performance assessment in patients receiving computer controlled propofol and manually controlled remifentanil infusions for minor surgery. British Journal of Anaesthesia 90, No. 6. pp. 737-741.

Åström, K.J.; Wittenmark, B. (1994). Adaptive Control (2nd ed.). Addison-Wesley Publishing Company, Inc.

Bressan, N.; Moreira, A.P.; Amorim, P.; Nunes, C.S. (2009). Target controlled infusion algorithms for anesthesia: Theory os practical implementation. Engineering in Medicine and Biology Society, EMBC 2009. Annual International Conference of the IEEE, pp. 6234-6237.

Dumont, G.A.; Martinez, A.; Ansermino, J.M. (2009). Robust control of depth of anesthesia. International Journal of Adaptive Control and Signal Processing, No. 23. pp. 435454.

Furutani, E.; Sawaguchi, Y.; Shirakami, G.; Araki, M.; Fukuda, K. (2005). A hypnosis control system using a model predictive controller with online identification of individual parameters. Proceedings of the 2005 IEEE Conference on Control Applications. Toronto, Canada, August, pp. 28-31.

Gil, F.G. (2004). Sistema de ayuda a la toma de decisiones mediante logica fuzzy en anestesia intravenosa: modelo farmacocinetico/farmacodinamico del propofol. PhD. Thesis, Universidad de Murcia, España.

Ionescu, C.M.; De Keyser, R.; Torrico, B.C.; De Smet, T.; Struys, M.M.; Normey-Rico, J.E. (2008). Robust Predictive Control Strategy Applied for Propofol Dosing Using BIS as a Controlled Variable During Anesthesia. IEEE Transactions On Biomedical Engineering, Vol. 55, No. 9, pp. 2161-2170. 
Kazama, T.; Ikeda, K.; Morita, K.; Kikura, M.; Doi, M.; Ikeda, T.; Kurita, T.; Nakajima, Y. (1999). Comparison of the Effect-site keO s of Propofol for Blood Pressure and EEG Bispectral Index in Elderly and Younger Patients. Anesthesiology, Vol. 90, No. 6, June, pp. 1517-1527.

Liu, N.; Chazot, T.; Genty, A.; Landais, A.; Restoux, A.; McGee, K.; Laloë, P.A.; Trillat, B.; Barvais, L.; Fischler, M. (2006). Titration of Propofol for Anesthetic Induction and Maintenance Guided by the Bispectral Index: Closed-loop versus Manual Control. A Prospective, Randomized, Multicenter Study. Anesthesiology, No. 104. pp. 686-695.

Marsh, B.; White, M.; Morton N.; Kenny, G.N.C. (1991). Pharmacokinetic model driven infusion of propofol in children. Br J Anaesth., Vol. 67, pp. 41-48.

Morley, A., Derrick, J., Mainland, P., Lee, B. B. and Short, T. G. (2000). Closed loop control of anaesthesia: an assessment of the bispectral index as the target of control. Anaesthesia, 55. pp. 953-959.

Niño J.; De Keyser, R.; Syafiie, S.; Ionescu, C.; Struys, M.M. (2009). EPSAC-controlled anesthesia with online gain adaptation. International Journal of Adaptative Control and Signal Processing, No. 23, pp. 455-471.

Sawaguchi, Y.; Purutani, E.; Shirakami, G.; Araki, M.; Fukuda, K. (2003). A model predictive sedation control system under total intravenous anesthesia. Proceedings of the IEEE EMBS Asian-Pacific Conference on Biomedical Engineering, pp. 358- 359.

Sakai, T.; Matsuki A.; White P.F.; Giesecke A.H. (2000). Use of an EEG-bispectral closed-loop delivery system for administering propofol. Anaesthesiologica Scandinavica, 44. pp. 1007-1010.

Schnider, T.W.; Minto, C.F.; Gambus, P.L.; Anderson, C.; Goodale, D.B.; Shafer S.L.; Youngs, E.J. (1998). The Influence of method of administration and covariates on the pharmacokinetics of propofol in adult volunteer. Anesthesiology, Vol. 88, pp. 11701182.

Sigl, J.C.; Chamoun, N.G. (1994). An introduction to bispectral analysis for the electroencephalogram. Journal of Clinical Monitoring, Vol. 10, No.6, pp. 392-404.

Smith, C. (1972). Digital Computer Process Control. Intext Education Publishers. Scranton PA.

Sreenivas, Y.; Samavedham, L.; Rangaiah, G. P. (2008). Advanced Regulatory Controller for Automatic Control of Anesthesia. Proceedings of the 17th World Congress of The International Federation of Automatic Control, Seoul, Korea, July, pp. 6-11.

Sreenivas, Y.; Samavedham, L. Rangaiah, G. P. (2009). Advanced Control Strategies for the Regulation of Hypnosis with Propofol. Ind. Eng. Chem. Res., No. 48, pp. 3880-3897.

Struys, M.M.; De Smet, T.; Depoorter, B.; Versichelen, L.F.; Mortier, E.P.; Dumortier, F.J.; Shafer, S.L.; Rolly, G. (2000). Comparison of plasma compartment versus two methods for effect compartment-controlled target controlled infusion for propofol. Anesthesiology, Vol. 92, No. 2, pp. 399-406.

Struys, M.M.; De Smet, T.; Versichelen, L.F.; Van De Velde, S.; Van den Broecke, R.; Mortier, E.P. (2001). A Comparison of Closed-loop Controlled Administration of Propofol Using Bispectral Index as the Controlled Variable versus "Standard Practice" Controlled Administration. Anesthesiology, No. 95. pp. 6-17. 
Syafiie, S.; Niño, J.; Ionescu, C.; De Keyser, R. (2009). NMPC for Propofol Drug Dosing during Anesthesia Induction. L. Magni et al. (Eds.): Nonlinear Model Predictive Control, LNCIS, No. 384, pp. 501-509. 


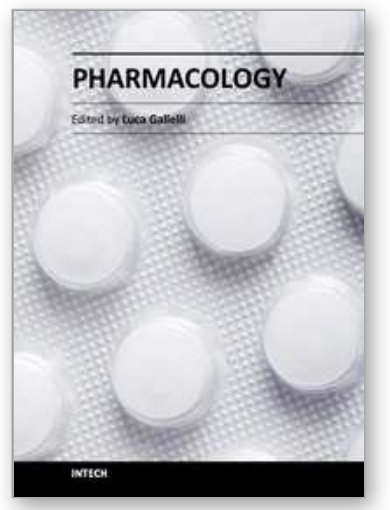

\author{
Pharmacology \\ Edited by Dr. Luca Gallelli
}

ISBN 978-953-51-0222-9

Hard cover, 720 pages

Publisher InTech

Published online 14, March, 2012

Published in print edition March, 2012

The history of pharmacology travels together to history of scientific method and the latest frontiers of pharmacology open a new world in the search of drugs. New technologies and continuing progress in the field of pharmacology has also changed radically the way of designing a new drug. In fact, modern drug discovery is based on deep knowledge of the disease and of both cellular and molecular mechanisms involved in its development. The purpose of this book was to give a new idea from the beginning of the pharmacology, starting from pharmacodynamic and reaching the new field of pharmacogenetic and ethnopharmacology.

\title{
How to reference
}

In order to correctly reference this scholarly work, feel free to copy and paste the following:

Santiago Torres, Juan A. Méndez, Héctor Reboso, José A. Reboso and Ana León (2012). Closed-Loop Control of Anaesthetic Effect, Pharmacology, Dr. Luca Gallelli (Ed.), ISBN: 978-953-51-0222-9, InTech, Available from: http://www.intechopen.com/books/pharmacology/closed-loop-control-of-anesthetic-effect

\section{INTECH}

open science | open minds

\section{InTech Europe}

University Campus STeP Ri

Slavka Krautzeka 83/A

51000 Rijeka, Croatia

Phone: +385 (51) 770447

Fax: +385 (51) 686166

www.intechopen.com

\section{InTech China}

Unit 405, Office Block, Hotel Equatorial Shanghai

No.65, Yan An Road (West), Shanghai, 200040, China 中国上海市延安西路65号上海国际贵都大饭店办公楼405单元

Phone: +86-21-62489820

Fax: +86-21-62489821 
(C) 2012 The Author(s). Licensee IntechOpen. This is an open access article distributed under the terms of the Creative Commons Attribution 3.0 License, which permits unrestricted use, distribution, and reproduction in any medium, provided the original work is properly cited. 